\title{
Nail dermoscopy (onychoscopy) is useful in diagnosis and treatment follow-up of the nail mixed infection caused by Pseudomonas aeruginosa and Candida albicans
}

\author{
Alicja Romaszkiewicz, Martyna Sławińska, Michał Sobjanek, Roman J. Nowicki \\ Department of Dermatology, Venereology and Allergology, Medical University of Gdansk, Gdansk, Poland \\ Adv Dermatol Allergol 2018; XXXV (3): 327-329 \\ DOI: https://doi.org/10.5114/ada.2018.76232
}

Nail dermoscopy (onychoscopy) is a non-invasive diagnostic tool helpful in differential diagnosis of nail apparatus pigmentation. In the Caucasian population melanocytic melanonychia occurs relatively rarely. More common causes (about 80\%) of dark nail pigmentation are haematomas and nail infections [1]. From the clinical point of view, it is important to distinguish between these conditions to avoid unnecessary biopsies. Onychoscopy is a simple, non-invasive tool which supports the clinical diagnosis. Dermoscopic features of nail pigmentation caused by melanocytic lesions (melanonychia) are described and also the dermoscopic pattern of the nail apparatus melanoma is established. In contrast, there are only a few reports of dermoscopic features of the nail pigmentation caused by infectious agents [2-4]. Similarly, dermoscopic features of mixed nail infections due to Pseudomonas aeruginosa (P. aeruginosa) and Candida albicans (C. albicans) have not been previously reported. We present two illustrative cases indicating the usefulness of dermoscopy in differential diagnosis of infectious and melanocytic nail pigmentation as well as its possible application in treatment follow-up of mixed nail infections.

Two unrelated, otherwise healthy, women (40- and 44-year-old) presented to the Department of Dermatology with dark pigmented, longitudinal band of the hand nails (Figures 1 A, B). The symptoms were observed for a few months. In patient 1, onychoscopy revealed irregular brown-green discolouration (Figure 1 C). In patient 2, onychoscopy revealed a mix of black, grey and yellow discolouration of the nail and the presence of scaly surface (Figure $1 \mathrm{D}$ ). In both cases the provisional diagnosis of nail infection was made. Microbiological examinations confirmed the presence of mixed $-C$. albicans and P. aeruginosa - infection. Both patients were treated with oral fluconazole (200 mg per week), topical ciprofloxacin (eye drops) and topical ciclopirox. After 1-month treatment, the narrowing of the streaks was visible (Figures $2 \mathrm{~A}, \mathrm{~B}$ ). After 3-month treatment, almost complete resolution of the pathological pigmentation of the nails was observed. The only visible alterations were onycholysis and subungual hyperkeratosis.

Recently, well-documented research on dermoscopic examination of melanonychia has been published. Also our Polish study confirmed the usefulness of onychoscopy in differential diagnosis of nail pigmentation (melanonychia/chromonychia) and evaluation of the nail apparatus melanoma [5]. In the majority of cases, melanocytic origin can be excluded based on clinical examination (colour and shape of the lesion). Some cases of chromonychia - manifesting as a longitudinal dark pigmented streak - may mimic melanonychia. In these cases onychoscopy may give a clue to correct diagnosis.

Various infectious agents may cause chromonychia - most commonly dermatophytes and P. aeruginosa. Ohn et al. [2] performed a comparative study of fungal melanonychia (FM) and other causes of melanonychia. The results showed that FM has distinct dermoscopic features facilitating differential diagnosis with melanocytic pigmentation including: the presence of yellow colour, white/yellow streaks, subungual keratosis and the absence of longitudinal patterns as well as pseudoHutchinson sign. A classical clinical manifestation of $P$. aeruginosa nail infection is described as the green nail syndrome. However, observed nail discolouration may also be greenish-black, bluish-grey, greenish-brown and greenish-yellow [6]. Fungal melanonychia caused by C. albicans is rare with only a few cases reported so far $[7,8]$. To the best of our knowledge, onychoscopic features of this condition have not been reported to date. Based on the presented cases it is difficult to conclude if the presence of black, green or yellowish pigmentation

Address for correspondence: Alicja Romaszkiewicz, Department of Dermatology, Venereology and Allergology, Medical University of Gdansk, 1a Kliniczna St, 80-402 Gdansk, Poland, phone: +48 5434925 81, e-mail: romaszkiewicz@gumed.edu.pl Received: 21.03.2017, accepted: 1.10.2017. 

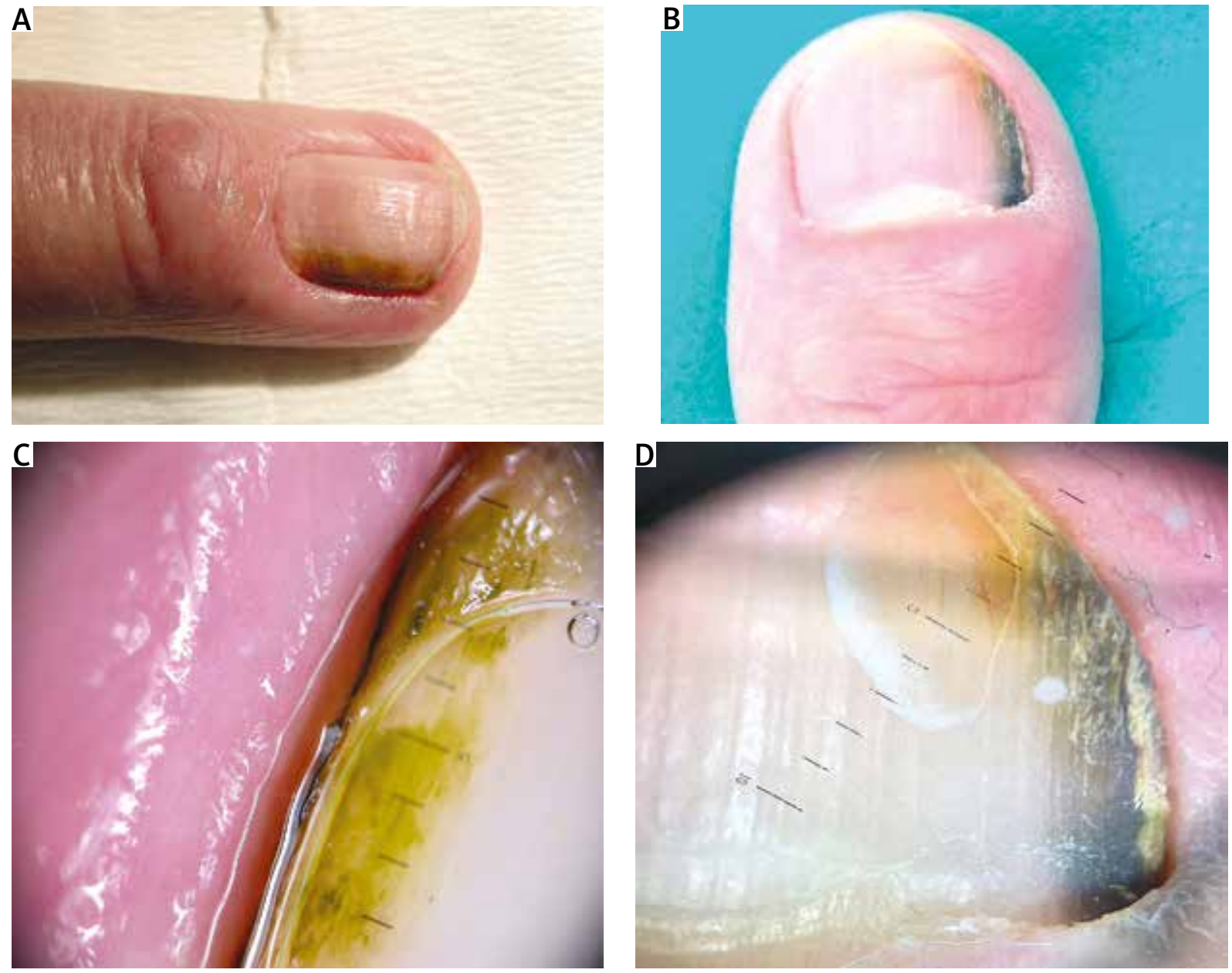

Figure 1. A - Dark streak on the lateral part of the middle fingernail (patient 1), B - dark streak on the lateral part of the left thumbnail (patient 2), C - onychoscopy shows irregular brown-green discolouration (patient 1), D - onychoscopy shows a mix of black, grey and yellow discolouration of the nail and the presence of scaly surface (patient 2)
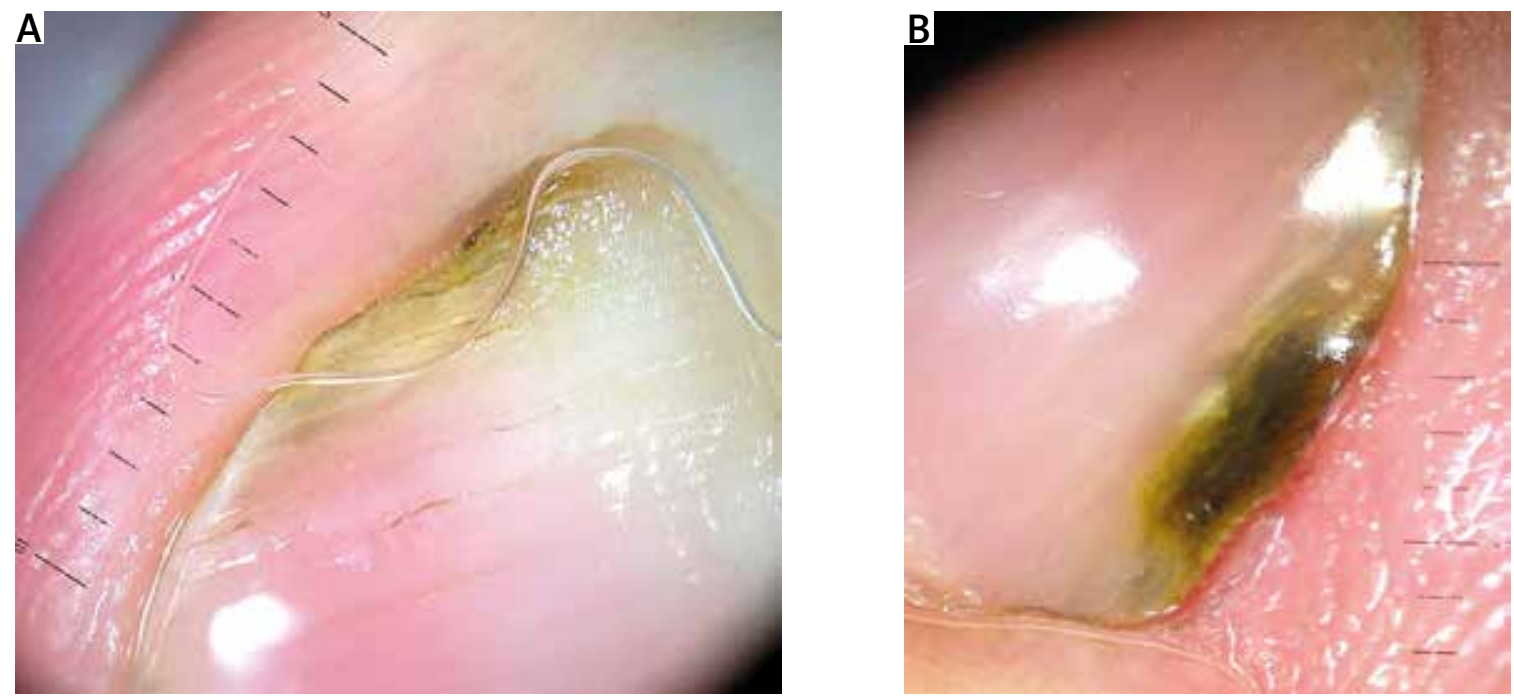

Figure 2. A - Follow-up onychoscopy performed 1 month after the treatment (patient 1), B - follow-up onychoscopy performed 1 month after the treatment (patient 2) 
was a result of $C$. albicans or $P$. aeruginosa as both pathogens may be responsible for such nail plate discolouration seen macroscopically. Further onychoscopic observations are required to assess the utility of dermoscopy in differential diagnosis of isolated/combined nail infections caused by P. aeruginosa and C. albicans.

The value of onychoscopy in monitoring of the treatment efficacy of nail infections is a novel observation. Treatment of mixed nail infections due to P. aeruginosa and $C$. albicans may be difficult. There are several treatment options including topical and oral drugs as well as nail plate avulsion $[6,9]$. The presented cases strongly suggest that less advanced infections may be treated without the surgical removal of the nail plate.

In conclusion, onychoscopy is a very useful tool facilitating differential diagnosis of various nail conditions, including mixed infections.

\section{Acknowledgments}

The article is financed by Polish Ministry of Scienceand Higher Education grant 02-0066/07/253.

\section{Conflict of interest}

The authors declare no conflict of interest.

\section{References}

1. Sobjanek M, Michajlowski I, Wlodarkiewicz A, et al. Longitudinal melanonychia in a northern Polish population. Int J Dermatol 2014; 53: e41-2.

2. Ohn J, Choe YS, Park J, et al. Dermoscopic patterns of fungal melanonychia: a comparative study with other causes of melanonychia. J Am Acad Dermatol 2017; 76: 488-93.e2.

3. Piraccini BM, Balestri R, Starace M, et al. Nail digital dermoscopy (onychoscopy) in the diagnosis of onychomycosis. J Eur Acad Dermatol Venereol 2013; 27: 509-13.

4. Kilinc Karaarslan I, Acar A, Aytimur D, et al. Dermoscopic features in fungal melanonychia. Clin Exp Dermatol 2015; 40: 271-8.

5. Sobjanek M, Michajłowski I, Biernat W, et al. Clinical and dermoscopic features of nail apparatus pigmentations. Przegl Dermatol 2013; 100: 65-79.

6. Chiriac A, Brzezinski P, Foia L, Marincu I. Chloronychia: green nail syndrome caused by Pseudomonas aeruginosa in elderly persons. Clin Interv Aging 2015; 10: 265-7.

7. Cho E, Lee YB, Park HJ, et al. Fungal melanonychia due to Candida albicans. Int J Dermatol 2013; 52: 1598-600.

8. Finch J, Arenas R, Baran R. Fungal melanonychia. J Am Acad Dermatol 2012; 66: 830-41.

9. Nenoff P, Paasch U, Handrick W. Infections of finger and toe nails due to fungi and bacteria. Hautarzt 2014; 65: 337-48. 